\title{
Concern about Japan's unclear biotech regulations
}

\author{
To exploit genetics research, agencies need to have clearly defined responsibilities.
}

Sir - The Cartagena Protocol on Biosafety (www.biodiv.org/biosafety), formulated in 2000 , is concerned with standardizing the movement of genetically modified organisms (GMOs) across national boundaries. As of January 2003, more than 100 countries have signed and 41 ratified the protocol. Only nine more countries need to ratify the protocol for it to become internationally and universally binding.

Further discussions are needed to reach agreement on the details of each article within the protocol. Article 18, which I discuss here, concerns the handling, transport, packaging and identification of living modified organisms (as GMOs are legally termed) across boundaries. Because the protocol seems likely to be ratified soon, we are very close to requiring internationally standardized documentation and instruction for transboundary movements of these organisms.

Global scientific communities need to pay careful attention to future practices, rules and standards in international movements of these organisms, even if they are being used only for research. We must, of course, have a proper method for considering potential risks to the environment and human health in handling, transport, packaging and identification. Also, it is vital to avoid confusion, unfounded fear and consequent negative perceptions of biotechnology associated with genetic engineering.

There is particular concern among Japanese scientists and industry about the present status of legislation, proposed by the government, on research and development (R\&D) and industrial uses of living modified organisms, in the light of the biosafety protocol. Although commercial imports are unlikely to suffer from any new laws passed to meet consumer demands and concern, it may be impossible to regulate $\mathrm{R} \& \mathrm{D}$ and industrial applications, because of confusion about administrative responsibility and inadequate numbers of inspectors. Establishing comprehensive and workable rules under the protocol is urgent to avoid delays to R\&D and commercial activities, which would set Japan back further in relation to North America and Europe.

So far, there has been no commercial cultivation of GM crops in Japan, although government-approved GM cultivars could be planted and sold to consumers without major regulations. Public concern in Japan discourages interest in commercial crop cultivation R\&D. Work that has been carried out by academic research institutions and the private sector, and past investment in genetic engineering, may be wasted because of these concerns and the impending regulations.

Japan has less experience in field evaluation of GM crops than countries in the European Union; there have been four times more evaluations in Italy and eight times more in France, where commercialization is unpopular but research is tolerated (www.olis.oecd.org/biotrack.nsf). In commercial cultivation of GM crops, Japan lags behind other Asian countries. India and China have far more experience at the local farmers' level, with thousands of hectares of GM crops and much accumulated knowledge and experience for future development of crop technology and associated risk management.

Although there is high-quality basic research on plant molecular biology and genetic engineering in Japan, these valuable results have remained unexploited because of regulations and lack of support systems for implementing the environmental release of GM plants or a biosafety assessment. Japan needs regulatory agencies that have clear responsibilities, and clear, workable risk-management schemes in R\&D institutions.

Kazuo N. Watanabe

Gene Research Centre, University of Tsukuba, 1-1-1 Tennoudai, Tsukuba, Ibaraki 305-8572, Japan

\section{Reviewing should be shown in publication list}

Sir-M. H. Dominiczak's comments on peer review in Correspondence (Nature 421,$111 ; 2003$ ) are a useful reminder that this mainly secret activity is essential for the quality of scientific publications to be kept as high as possible. High-quality reviewing is time-consuming and in some ways is comparable to co-authorship, supervision and teaching, often giving rise to highly influential scientific debate. Yet it is almost without tangible reward, other than the private satisfaction of a job well done or the thanks of the author to an anonymous referee in the small print.

At present, very few scientific journals pay their reviewers. More substantial financial rewards are unlikely to improve the quality of reviews, and are too costly for most journals, as discussed in your News Feature "Publish, and be damned..." (Nature 419, 772-776; 2002). For many years, as your feature noted, some journals have published lists of their referees, and the American Geophysical Union runs a scheme to honour their best reviewers. Yet although these initiatives are welcome, they do not provide lasting motivation.

Scientists often briefly mention their reviewing activity in their $\mathrm{CVs}$, listing the journals and frequency of reviews. We propose that these qualifications should be made a standard part of the CV as well as of research units' annual reports. This offers relevant information about scientific status, acquaintance with the literature, and willingness to offer free advice, which in itself is of considerable relevance to grant applications and in appointments. The quality of the journals and the frequency of reviews would be a measure of distinction, as editors use good reviewers most often. Our proposal could gradually improve the quality and status of reviewing - assuming that journals would be willing to verify reviewers' figures and would provide a more enduring incentive for people to participate in this otherwise often frustrating duty.

Torben Clausen, Ole Bækgaard Nielsen Department of Physiology, University of Aarhus, DK-8000 Aarhus C, Denmark

\section{Flying into history}

Sir - The caption of your Commentary picture (Nature 421, 16; 2003) suggests that Orville Wright made the first powered flight in 1903. This is not true, although Heilbron's and Bynum's text, on the previous page, was correct. The Wright brothers - influenced by the nonpowered gliders of Otto Lilienthal, who flew more than $300 \mathrm{~m}$ in 1894 - were the first to achieve the important conjunction of four criteria with their 260-m flight: it was manned, powered, heavier-than-air and (to some degree) controlled.

Earlier pioneers set records by meeting some of these criteria. In 1890 Clément Ader made the first manned, powered, heavier-than-air flight, of $50 \mathrm{~m}$, in his bat-winged monoplane. Henri Giffard's steam-powered airship covered $27 \mathrm{~km}$ on the first manned and powered flight, in 1852. Balloonists Jean-François Pilâtre de Rozier and François d'Arlandes were, in 1783 , the first men to fly. And if they were as fashionably bewigged as the occasion demanded, their 9-km ride must also have been the first manned, powdered flight. Jürgen Schmidhuber

Dalle Molle Institute of Artificial Intelligence, Galleria 2, 6928 Manno (Lugano), Switzerland 